\title{
Association and Dissociation of Optimal Noise and Input Impedance for Low-Noise Amplifiers
}

Johansen, Daniel Højrup; Sanchez-Heredia, Juan D.; Zhurbenko, Vitaliy; Ardenkjær-Larsen, Jan Henrik

Published in:

IEEE Transactions on Microwave Theory and Techniques

Link to article, DOI:

10.1109/TMTT.2018.2869603

Publication date:

2018

Document Version

Peer reviewed version

Link back to DTU Orbit

Citation (APA):

Johansen, D. H., Sanchez-Heredia, J. D., Zhurbenko, V., \& Ardenkjær-Larsen, J. H. (2018). Association and Dissociation of Optimal Noise and Input Impedance for Low-Noise Amplifiers. IEEE Transactions on Microwave Theory and Techniques, 66(12), 5290 - 5299. https://doi.org/10.1109/TMTT.2018.2869603

\section{General rights}

Copyright and moral rights for the publications made accessible in the public portal are retained by the authors and/or other copyright owners and it is a condition of accessing publications that users recognise and abide by the legal requirements associated with these rights.

- Users may download and print one copy of any publication from the public portal for the purpose of private study or research.

- You may not further distribute the material or use it for any profit-making activity or commercial gain

- You may freely distribute the URL identifying the publication in the public portal 


\title{
Association and Dissociation of Optimal Noise and Input Impedance for Low-Noise Amplifiers
}

\author{
Daniel H. Johansen ${ }^{\circledR}$, Juan D. Sanchez-Heredia ${ }^{\circledR}$, Vitaliy Zhurbenko ${ }^{\circledR}$, and Jan H. Ardenkjær-Larsen ${ }^{(1)}$
}

\begin{abstract}
For magnetic resonance imaging (MRI) receive coil arrays, an ideal low-noise amplifier (LNA) is noise matched while exhibiting a high-input reflection coefficient of unity or slightly higher. For this purpose, we present a design approach allowing to manipulate the optimal noise impedance and input impedance. The method is based on noise and S-parameters, hence technology independent. As an example, the method is used to design an LNA for MRI receive coil arrays operating at $32.1 \mathrm{MHz}$. The design demonstrates the highest coil decoupling published so far of $54 \mathrm{~dB}$. The measured noise figure of $0.44 \mathrm{~dB}$ is also better than other published designs. The measured gain is $22 \mathrm{~dB}$ with a 1-dB compression point of $-\mathbf{1 4 . 5} \mathrm{dBm}$. The power consumption is $81 \mathrm{~mW}$. We expect this method to enable better MRI receive coil array designs resulting in lower examination time and cost due to higher quality images.
\end{abstract}

Index Terms-Biomedical imaging, design methodology, gain, impedance matching, low-noise amplifiers (LNAs), magnetic resonance, noise figure, optimized circuit design, scattering parameters.

\section{INTRODUCTION}

$\mathbf{L}$ OW-NOISE amplifiers (LNAs) are used in a plethora of applications involving the reception of small signals. In applications such as single surface or volume coils for magnetic resonance imaging (MRI), a classical noise matched (CNM) LNA is usually employed [1]. For wireless communications and nuclear magnetic resonance (NMR) spectroscopy probes, it is preferred to achieve a simultaneous noise and input match (SNIM). In the field of MRI receive coil arrays, a high-input reflection coefficient, noise matched (HRNM) LNA is required [2]. Hence, within the field of magnetic resonance, three cases of the LNA design are required: 1) SNIM for NMR spectroscopy probes; 2) CNM for MRI single/volume coils; and 3) HRNM for MRI coil arrays.

A general MRI system utilizes two orthogonal magnetic fields: a static field, called the main field, and a radio

Manuscript received March 1, 2018; revised May 18, 2018 and July 16, 2018; accepted August 19, 2018. This work was supported in part by the Danish National Research Foundation under Grant DNRF124. (Corresponding author: Daniel H. Johansen.)

D. H. Johansen, J. D. Sanchez-Heredia, and V. Zhurbenko are with the Department of Electrical Engineering and the Center for Hyperpolarization in Magnetic Resonance and Electromagnetic Systems, Technical University of Denmark, 2800 Kongens Lyngby, Denmark (e-mail: dhjo@elektro.dtu.dk).

J. H. Ardenkjær-Larsen is with the Department of Electrical Engineering and the Center for Hyperpolarization in Magnetic Resonance and Electromagnetic Systems, Technical University of Denmark, 2800 Kongens Lyngby, Denmark, and also with GE Healthcare, 2800 Kongens Lyngby, Denmark (e-mail: jhar@elektro.dtu.dk).

Digital Object Identifier 10.1109/TMTT.2018.2869603

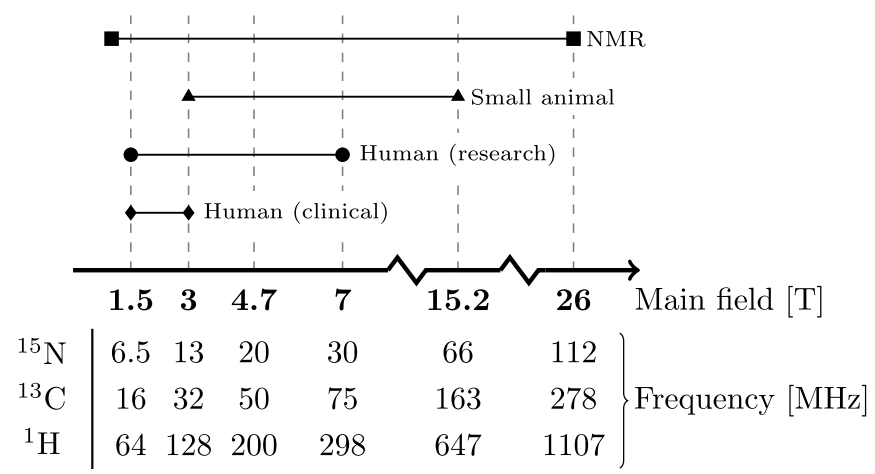

Fig. 1. Overview of the main field and corresponding operating frequency of three commonly used nuclei when used for different MR applications.

frequency (RF) field oscillating at the resonance frequency of the nuclei of interest (the Larmor frequency). The following description of magnetic resonance is very simplified. As a sample or a patient is placed in the main field, their nuclei become polarized (partially aligned with the main magnetic field). The RF field, if applied at the Larmor frequency, brings the nuclei out of equilibrium. When the RF field is turned off the nuclei precess back toward equilibrium producing an RF field at their Larmor frequency. Hence, the RF field is recorded by a receive coil and the signal is subsequently amplified by the LNA. The Larmor frequency is described by the product of the gyromagnetic ratio of the nuclei and the strength of the static magnetic field. Thus, the frequency of operation varies depending on the nucleus of interest and the main field strength. An overview of the operating frequency of three nuclei, along with four common applications, is shown in Fig. 1 in relation to the main field strength. The most commonly imaged nuclei are hydrogen $\left({ }^{1} \mathrm{H}\right)$, having a gyromagnetic ratio of approximately $42 \mathrm{MHz} / \mathrm{T}$. Other nuclei, such as carbon-13 $\left({ }^{13} \mathrm{C}\right)$, exhibit a much lower gyromagnetic ratio. Hence, the operating frequency of an MRI system varies from a few $\mathrm{MHz}$ up to approximately $1 \mathrm{GHz}$. As a consequence, not only must the design method be applicable to the three cases but also in a wide range of discrete frequencies. This is not the same as requiring a large bandwidth of the LNA, as the bandwidth in MRI is limited between approximately 50 and $100 \mathrm{kHz}$, depending on static field strength, nucleus, and imaging pulse sequence used [3], [4].

The method for obtaining SNIM is well described for a range of applications and is based on adding feedback to manipulate the relationship between the optimal noise 
match and input impedance [5]-[10]. For narrowband applications, inductive source degeneration is often used in a common-source topology [11]-[14]. Series feedback offers the advantage of not degrading the noise performance of the LNA [15]-[17]. Parallel feedback techniques are often employed for wideband applications. In most cases, the optimal noise match is shifted by changing the amount of feedback [5], [18]-[20].

The CNM LNA is thoroughly described in many textbooks [21], [22], with the earliest description dating back to the 1960s [23], [24]. Classically, the input is noise matched to achieve the lowest noise, and the output is gain matched. The resulting input impedance may exhibit a high reflection coefficient, which may be unacceptable for some applications.

In the low-frequency domain, HRNM LNAs are used in many instrumentation applications [25]. The design and review of low-frequency HRNM LNAs are described in depth by Levinzon [25]. A primary focus is on reducing flicker noise. Since flicker noise is not usually a problem in RF LNAs, much of this procedure is not very relevant at higher frequencies. In [26], a method primarily for SNIM is described. It is based on the same theory as this paper. Hence, it could be used for HRNM designs. However, Ciccognani et al. [26] assume ideal reactive input and output circuits and also state that the case of negative input resistance is not considered. In the case of LNAs for MRI receive coil arrays, little has thus far been published. Johansen et al. [27] describe a design which assumes that the input impedance of a field effect transistor is sufficiently high that an appropriately chosen inductor in the biasing of the transistor combined with a series capacitor yields an acceptable input impedance and noise performance. However, neither the input impedance nor the noise performance is optimal. A $50-\Omega$ transmission line equivalent circuit is placed in the input of the LNA to transform the input impedance to an acceptable value while the $50-\Omega$ noise performance is constant.

This paper defines the entire range of design cases and describes a design method applicable for the cases described earlier. The method also works for intermittent steps between the cases of SNIM, CNM, and HRNM. These cases are dubbed design by association, equilibrium, and dissociation. The method uses S-parameters (generally, any set of linear parameters is sufficient as long as their transformation to S-parameters is well defined) and noise parameters, and is thus technology agnostic. Even though the method works for all cases, it is mostly relevant for designs by dissociation. This paper is, to the best of our knowledge, the first to propose a method for complete dissociation. Even stable negative input resistance is possible. Hence, this method can also be used for negative resistance/impedance converters [28]-[31]. Using the presented method, an LNA for a $32.1-\mathrm{MHz}\left({ }^{13} \mathrm{C}\right.$ at $\left.3 \mathrm{~T}\right)$ MRI receive coil array is implemented showing better decoupling, noise figure, and power consumption than the other published LNA designs while maintaining an acceptable gain and linearity performance.

\section{Definitions and Problem Formulation}

The LNA is divided into three two-ports as seen in Fig. 2. The input circuit, transistor, and output circuit are described by

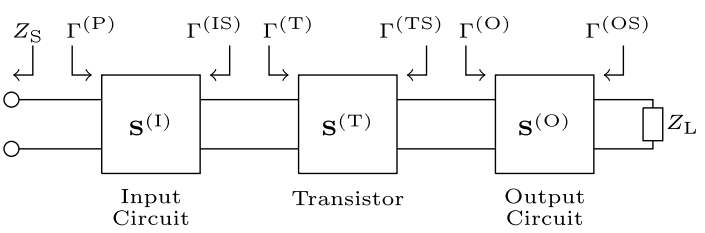

Fig. 2. Overview of the LNA which is divided into three two-ports: input circuit, transistor, and output circuit.

their corresponding S-parameter matrices, $\mathbf{S}^{(\mathrm{I})}, \mathbf{S}^{(\mathrm{T})}$, and $\mathbf{S}^{(\mathrm{O})}$, respectively. The terms impedance and reflection coefficient are used interchangeably since their conversions are well defined. To define the concepts of association, equilibrium, and dissociation the power wave reflection coefficient is used [32]. The equilibrium coefficient is defined as

$$
\rho_{\mathrm{E}}=\left|\frac{Z_{50 \Omega}^{(\mathrm{T})}-Z_{\mathrm{opt}}^{(\mathrm{T}) *}}{Z_{50 \Omega}^{(\mathrm{T})}+Z_{\mathrm{opt}}^{(\mathrm{T})}}\right|
$$

where $Z_{50 \Omega}^{(\mathrm{T})}$ is the input impedance of the transistor when it is loaded by $50 \Omega$. $Z_{\mathrm{opt}}^{(\mathrm{T})}$ is the optimal source impedance of the transistor that minimizes the noise figure. The asterisk signifies the complex conjugate. The equilibrium coefficient is principally a figure of merit of the transistor describing the inherent mismatch between the optimal noise impedance and the input impedance of the transistor when terminated by $50 \Omega$. The noise-input impedance coefficient is defined as

$$
\rho=\left|\frac{Z^{(\mathrm{P})}-Z_{\mathrm{opt}}^{(\mathrm{P} *}}{Z^{(\mathrm{P})}+Z_{\mathrm{opt}}^{(\mathrm{P})}}\right|
$$

where $Z^{(\mathrm{P})}$ is the input impedance of the LNA and $Z_{\mathrm{opt}}^{(\mathrm{P})}$ is the optimal noise impedance at the input of the LNA. The noise-input impedance coefficient is a requirement specified by the designer and is thus defined at the input of the LNA. The design target for an LNA generally spans the region between the three cases:

1) Association: $\rho<\rho_{\mathrm{E}}$, decreasing the mismatch between the optimal noise and input impedance. This also includes SNIM where the input impedance is equal to the complex conjugate of the optimal noise impedance, $Z_{\mathrm{P}}=Z_{\mathrm{opt}}^{(\mathrm{P})^{*}}$ resulting in $\rho=0$.

2) Equilibrium: $\rho=\rho_{\mathrm{E}}$, when nothing is explicitly done to alter the mismatch between the optimal noise and input impedance.

3) Dissociation: $\rho>\rho_{\mathrm{E}}$, when the mismatch of the optimal noise and input impedance is increased. In the case of LNAs for MRI receive coil arrays, $\rho \approx 1$ as this maximizes the potential decoupling between array elements.

The input reflection coefficient of the transistor and LNA are traditionally described as

$$
\begin{aligned}
& \Gamma^{(\mathrm{T})}=S_{11}^{(\mathrm{T})}+\frac{S_{12}^{(\mathrm{T})} S_{21}^{(\mathrm{T})} \Gamma^{(\mathrm{O})}}{1-S_{22}^{(\mathrm{T})} \Gamma^{(\mathrm{O})}} \\
& \Gamma^{(\mathrm{P})}=S_{11}^{(\mathrm{I})}+\frac{S_{21}^{(\mathrm{I})} \Gamma^{(\mathrm{T})}}{1-S_{22}^{(\mathrm{I})} \Gamma^{(\mathrm{T})}}
\end{aligned}
$$

respectively. Given a passive output circuit, the impedance looking into the output circuit toward the load is defined within the complex unit circle, $\left|\Gamma^{(\mathrm{O})}\right| \leq 1$. Because (3) and (4) 


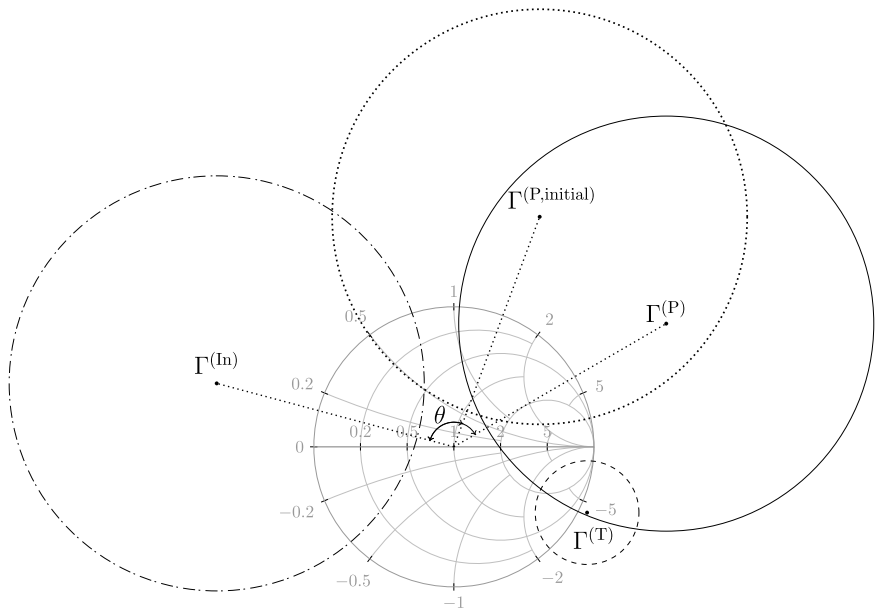

Fig. 3. Simulated transistor impedance circle (dashed), noise matched impedance circle (dashed dotted), initial input impedance (dotted), and final LNA input impedance circle (solid).

constitute a Möbius Transformation, having the basic property of transforming a circle to a circle, $\Gamma^{(\mathrm{T})}$ and $\Gamma^{(\mathrm{P})}$ are also described by a circle [33]. This case arises when the reverse isolation and the gain of the transistor is nonzero, $\left|S_{12}^{(\mathrm{T})}\right|>0$ and $\left|S_{21}^{(\mathrm{T})}\right|>0$. Hence, when feedback is present in the transistor, the input impedance of the transistor can be adjusted by the impedance of the output circuit.

If the gain of the transistor is high, the noise figure of the LNA can be assumed constant, regardless of loss in the output circuit (as long as the loss is lower than the gain of the transistor). The required $\Gamma^{(\mathrm{O})}$, when the input circuit and LNA input impedance are known, is given by

$$
\Gamma^{(\mathrm{O})}=\frac{\Gamma^{(\mathrm{P})}-S_{11}^{(\mathrm{IT})}}{S_{22}^{(\mathrm{IT})}\left(\Gamma^{(\mathrm{P})}-S_{11}^{(\mathrm{IT})}\right)+S_{12}^{(\mathrm{IT})} S_{21}^{(\mathrm{IT})}}
$$

where superscripted (IT) indicates the combined S-parameters of the input circuit and transistor. Hence, given a certain input circuit and transistor configuration, (5) describes the required impedance of the output circuit looking toward the load. Using the circle equation, the input impedance can be plotted as a function of $\Gamma^{(\mathrm{O})}$ when a given input circuit and the transistor is realized, as seen in Fig. 3 (detailed in Section III). The main issue is that $\Gamma^{(\mathrm{O})}$ may become larger than unity, depending on the input circuit and transistor. In this paper, only the inherent feedback present in the transistor is used. Hence, no additional feedback is added. The usage of feedback for the LNA design is well described in other works (see [8]). In terms of noise parameters, only the optimal noise impedance is directly manipulated in this paper by the input circuit. The choice of biasing voltage/current of the transistor can have an enormous impact on all noise parameters which is also described in depth in other works (see [21]). As mentioned, the main problem of (5) is choosing a proper input circuit resulting in a realizable passive output circuit when a certain $\Gamma^{(\mathrm{P})}$ is required.

\section{Design by Dissociation}

This section presents the design strategy and exemplifies the implementation by designing an LNA for an MRI receive coil array. Since MRI LNAs are inherently narrowband, the design method is applied at a single frequency. A brief discussion of applying the design method for broadband applications is found in the Appendix. The design strategy is divided into six steps.

Step 1) Transistor Selection: Find an appropriate transistor by determining the required optimal noise figure, reverse isolation, and gain. Plot (3) to confirm if the desired degree of association or dissociation is possible. Determine the S-parameters of the transistor at a given bias and supply voltage.

Step 2) Match for Optimal Noise: Design the input matching circuit such that the optimal noise figure impedance is transformed to the required impedance, $Z^{(\mathrm{IS})}=Z_{\mathrm{opt}}^{(\mathrm{T})}$. The required impedance can, principally, be anything but is normally $50 \Omega$. Furthermore, biasing and stability should be considered.

Step 3) Add Series Transmission Line to Input: To ensure a passive output circuit, the input impedance circle determined by (3) and (4) needs to be transformed to cover the required input impedance. Adding a series transmission line, with a characteristic impedance equal to the required optimal noise impedance, rotates the input impedance circle while the optimal noise match remains constant.

Step 4) Design Output Circuit: First, determine $\Gamma^{(\mathrm{O})}$ such that $\Gamma^{(\mathrm{P})}$ results in the required input reflection coefficient by applying (5). Second, add a loss to the output circuit to ensure stability. Also, investigate the frequency response to determine possible stability issues. Finally, the output circuit must also ensure that the output impedance of the preamplifier is matched to $Z_{\mathrm{L}}$.

Step 5) Determine Gain and Linearity Tradeoff: Since the gain and linearity of the LNA may be impaired by the choice of $\Gamma^{(\mathrm{O})}$ and length of the transmission line, iterate over Steps 1) and 4) to determine the gain and linearity tradeoff.

Step 6) Simplify Input Circuit: Since the implementation of a transmission line may be inconvenient, especially at lower frequencies, a Pi- or T-network equivalent can be utilized. The input thus consists of a noise match and discrete transmission line equivalent which may entail high losses (due to undesirable values). The entire input circuit can be further simplified to either a T- or Pi-network using the basic circuit theory.

Because the MRI array elements couple inductively, the impedance looking into the individual element's matching network should be large, particularly during the reception, to limit their coupling [2], [34]. The coupling between elements degrades the signal-to-noise ratio (SNR), making it difficult in practice to match all the elements, and increases noise correlation, which can be detrimental to parallel imaging. The combination of matching and decoupling circuits are described in [2], [35], and [36].

The requirements for the LNA is an optimal noise match at $50 \Omega$ with an inductive input impedance of approximately $360 \mathrm{nH}$. To ensure that the analog-to-digital converter of the MRI scanner is not saturated, the maximum allowable 


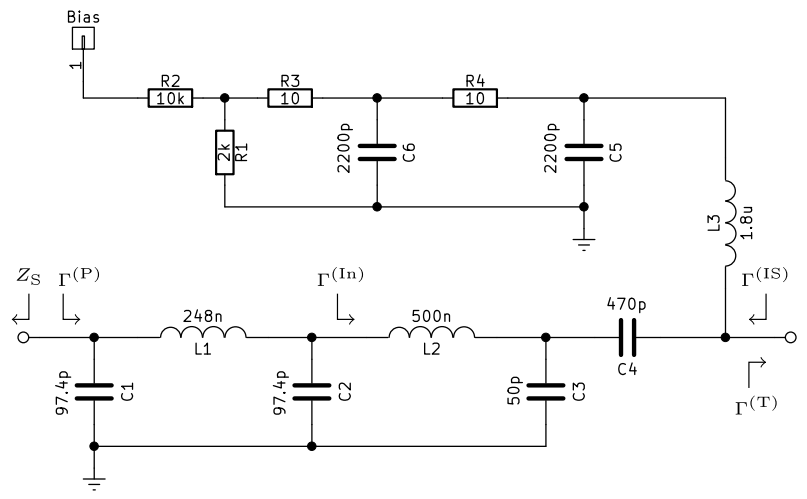

(a)

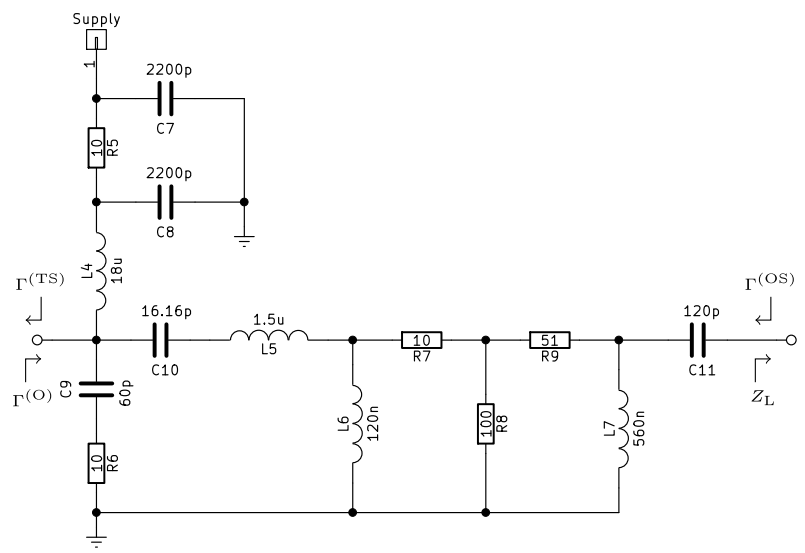

(b)

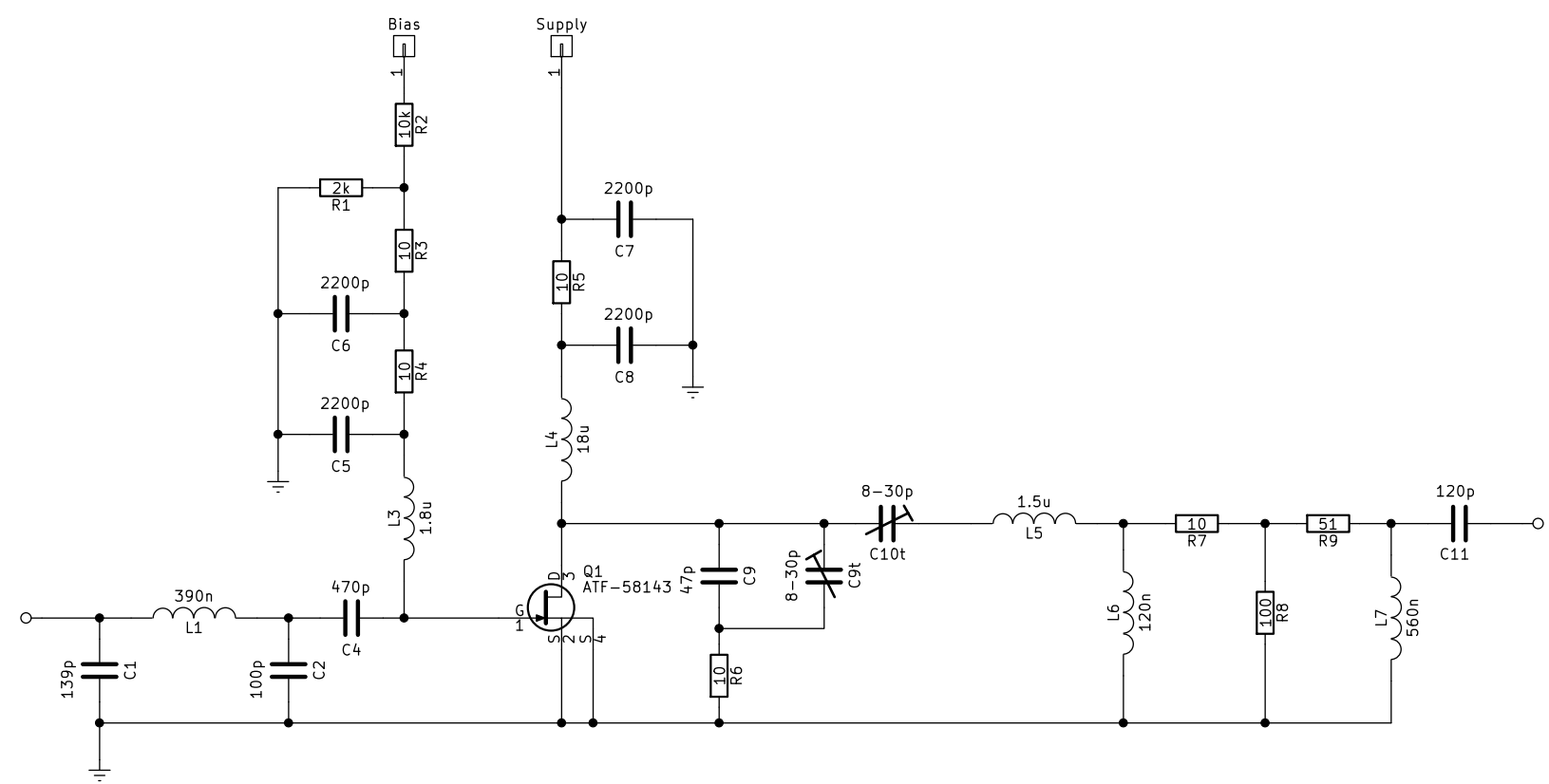

(c)

Fig. 4. Schematics used for the design and implementation of the LNA. (a) Input circuit used for analysis. Ideal transmission line is converted to a Pi-network (C1, C2, and L1). L2 and C3 perform optimal noise matching. (b) Output circuit used for analysis. R6 and C9 determine the degree of instability, while C10 and L5 determine the resonance of the negative resistance. R7, R8, and R9 form an attenuator to control the gain of the LNA. C11 and L7 match the output of the LNA to $50 \Omega$. (c) Schematic of the implemented LNA. Input circuit has been further simplified by combining the series transmission line and noise matching components into a Pi-network $(\mathrm{C} 1, \mathrm{C} 2$, and $\mathrm{L} 1)$. Output circuit is implemented using variable capacitors to control the degree of stability $(\mathrm{C} 9 \mathrm{t})$ and the notch where the input resistance becomes negative (C10t).

gain is $27 \mathrm{~dB}$. Finally, the LNA needs to be output matched to $50 \Omega$. The primary target is to maximize the decoupling between elements, which requires a negative input resistance of $-1.1 \Omega$ to compensate for the loss in the matching circuit.

In this example, the selection of transistor is based primarily on two parameters: the rated noise figure and cryogenic operation capability (even though it is not used at cryogenic temperatures in this paper). Furthermore, the MRI system features only a positive voltage supply. Hence, it decreases the necessary auxiliary circuitry to use a transistor, which requires a positive bias voltage. To satisfy the requirements outlined above, the enhancement mode pseudomorphic highelectron-mobility transistor Broadcom/Avago ATF58143 is chosen. It shows a minimum noise figure of $0.007 \mathrm{~dB}$ at $32.1 \mathrm{MHz}$ (extracted from the transistor model) with a bias voltage of $0.5 \mathrm{~V}$ and a supply voltage of $3 \mathrm{~V}$ [37]. We chose to use the well-known common-source topology for the design (with no inductive source degeneration). This further yields $Z_{11}^{(\mathrm{T})}=7-j 632 \Omega$ and an optimal noise impedance of $1403+j 729 \Omega$. The equilibrium coefficient is $\rho_{\mathrm{E}}=0.99$. The required input-noise coefficient is $\rho=1.014$. Since $\rho>\rho_{\mathrm{E}}$, dissociation is required. Additionally, the flicker noise corner frequency, extracted from the transistor model, is $1 \mathrm{MHz}$. Hence, the design does not require methods for lowering flicker noise.

The transistor input circle $\Gamma^{(\mathrm{T})}$ is calculated using (3) and (4), and the circle equation. Assuming a passive output circuit, the corresponding circle is seen in Fig. 3 (dashed circle) with a center in $(0.95 ;-0.47)$ and radius of 0.37 . This verifies that the transistor works for the degree of dissociation required.

The input circuit is shown in Fig. 4(a). The biasing inductor $\mathrm{L} 3$ together with $\mathrm{C} 5, \mathrm{R} 4$, and $\mathrm{C} 6$ constitute a low- $Q$ high-pass filter, with a resonance at $1.8 \mathrm{MHz}$, ensuring 
low-frequency stability. It was empirically discovered that if L1 is larger than approximately $2.2 \mu \mathrm{H}$ instability occurs. $\mathrm{L} 1$ has a rated internal resistance of $1 \Omega$. The high-pass filter exhibits an impedance at $32.1 \mathrm{MHz}$ of $1.4+j 361 \Omega$ adding noise corresponding to $0.027 \mathrm{~dB}$. The final components in the feeding part of the input circuit are $R 1$ and $R 2$. They perform a voltage division such that the supply voltage of $3 \mathrm{~V}$ is decreased to approximately $0.5 \mathrm{~V} . R 1$ and $R 2$ further ensure that the high-pass filter is loaded with a high impedance. Hence, $R 1$ and $R 2$ buffers the feeding network such that the resonance of the high-pass filter is not dependent on the network connected to the bias pin.

The second part of the input circuit is the noise match. $\mathrm{C} 4$ is a dc block. C3 and L2 match the optimal noise impedance to $50 \Omega$. The noise added by these three components is $0.011 \mathrm{~dB}$. The resulting noise matched impedance is $Z^{(\mathrm{In})}=$ $0.4-j 12 \Omega$ when the transistor is loaded with $50 \Omega$. The resulting noise matched input circle $\Gamma^{(\mathrm{In})}$ has its center in $(-1.7 ; 0.45)$ with radius 1.5 . It is also plotted in Fig. 3 (dashed dotted circle). This further yields the maximum dissociation $\rho_{\max }=3.26$ and the minimum association $\rho_{\min }=0.26$, which is realizable with a passive output circuit.

$\mathrm{C} 1, \mathrm{~L} 1$, and $\mathrm{C} 2$ is the discrete equivalent of a transmission line with an electrical length of $47.7^{\circ}$ and a characteristic impedance of $50 \Omega$. The transmission line rotates the center of the noise matched circle $\Gamma^{(\mathrm{In})}$ to the corresponding angle of the required input impedance. The resulting LNA input circle $\Gamma^{(\mathrm{P} \text {,initial })}$, with center $(0.6 ; 1.6)$ and radius 1.5 , is also seen in Fig. 3 (dotted circle). The electrical length of the transmission line of $47.7^{\circ}$ is used as the initial guess for starting the optimization procedure involving the determination of $\Gamma^{(\mathrm{O})}$, gain, and the linearity of the LNA. The transmission line equivalent circuit adds another $0.033-\mathrm{dB}$ noise. This yields a total noise of $0.071 \mathrm{~dB}$. The transmission line length of $47.7^{\circ}$ results in a required $Z^{(0)}=210+j 24 \Omega$. Through theoretical and practical iterations, an acceptable tradeoff between gain, linearity, and the simplicity and practicality of the output circuit resulted in a transmission line length of $67.5^{\circ}$. The resulting, and final, $\Gamma^{(\mathrm{P})}$ circle is shown in Fig. 3 (solid circle) with center $(1.5 ; 0.9)$ and radius 1.5 . Hence, $Z^{(\mathrm{O})}=$ $34+j 20 \Omega$.

The output circuit is seen in Fig. 4(b) and is made of four parts: feed, matching for $Z^{(\mathrm{O})}$, a T-network attenuator, and matching to $Z_{\mathrm{L}}$. The feed network consists of $\mathrm{L} 4, \mathrm{C} 7, \mathrm{C}$, and R5. L4 is the feed inductor. C7, C8, and R5 are supply filters.

The matching for $Z^{(0)}$ is performed by C9, C10, L5, L6, and R6. R6 and C9 form a low-pass filter and ensure stability at higher frequencies. To be able to adjust $Z^{(\mathrm{O})}, \mathrm{C} 9$ is chosen such that, by varying its value, the input impedance of LNA can also be varied. C9 primarily influences the resistance of $Z^{(\mathrm{P})}$. C10 and L5 form a series resonance determining the bandwidth in which the LNA can exhibit a negative input resistance. When C10 and L5 are resonant, they are simply shorted. However, slightly off resonance C10 and L5 ensure that the input impedance of LNA is not negative. This is to better control the stability due to a negative input resistance requirement. This creates a "bump" in the frequency response

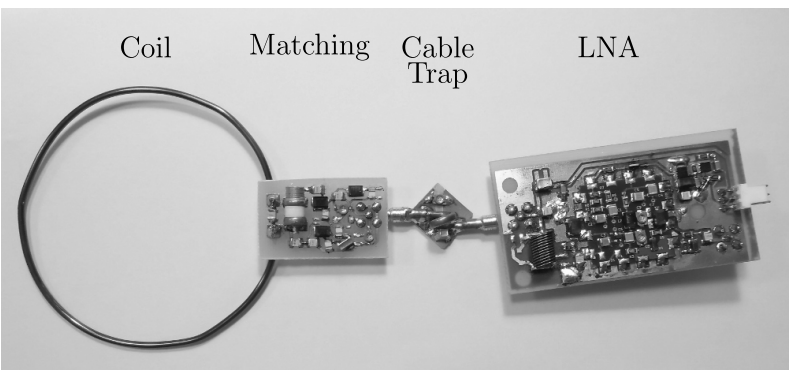

Fig. 5. Implemented setup. From left to right: MRI surface coil, matching and decoupling circuit, cable trap, and LNA. Diameter of the surface coil is $80 \mathrm{~mm}$.

of the input impedance where the negative resistance can occur, as will be shown later. Hence, by varying $\mathrm{C} 9$ and $\mathrm{C} 10$, the amount of negative input resistance and the frequency where the negative input resistance occurs can be controlled. L6 transforms the approximate $50-\Omega$ impedance looking into R7 toward the load toward the required $Z^{(\mathrm{O})}$.

To comply with the required $Z^{(\mathrm{O})}$, while matching $Z^{(\mathrm{OS})}$ to $50 \Omega$, adding loss to the output circuit is required to create the desired isolation between the two ports. The loss is added by resistors R7-R9. The loss is approximately $10 \mathrm{~dB}$. This yielded an acceptable gain of the LNA and isolation such that $\mathrm{C} 9$ and $\mathrm{C} 10$ can be varied without changing $Z^{(\mathrm{OS})}$ significantly. The total added noise by the output circuit is $0.01 \mathrm{~dB}$. Thus, the noise figure of the LNA is $0.081 \mathrm{~dB}$.

L7 and C11 match the output of the LNA to approximately $50 \Omega$. C11 also acts as a dc block, because, in some cases, a dc voltage will be supplied by the MRI system.

The final step in the design procedure is to simplify the input circuit. Since the input circuit also ensures stability at low frequencies, this must still apply after simplification.

The implemented schematic is shown in Fig. 4(c). The input circuit is simplified by merging the noise matching components and transmission line into a single Pi-network $(\mathrm{C} 1, \mathrm{C} 2$, and L1). Variable capacitors are added to the output circuit to tune the output circuit's impedance accurately. Furthermore, parasitic coupling between inductors L1, L3, L4, and L6 has been added to the model, in the order of $-40 \mathrm{~dB}$, to properly simulate the impact of the layout on the performance of the LNA. This adds approximately $0.15-\mathrm{dB}$ of noise and introduces changes to the input impedance. However, the input impedance is easily corrected by the variable capacitors C9t and C10t.

The implemented setup is shown in Fig. 5. A coil with a diameter of $80 \mathrm{~mm}$ is used. Matching is done with a shunt-series topology as described in [36]. The matching circuit also incorporates an active transmitter detuning to not to distort the transmit field or damage the LNA. A cable trap is also implemented to attenuate common-mode signals on the coaxial cables.

\section{RESUlTS}

The simulated and measured input and output impedances are seen in Fig. 6. The output impedance exhibits a voltage standing wave ratio of less than 1.15 at $32.1 \mathrm{MHz}$ for both the simulated and measured results. The input impedance 


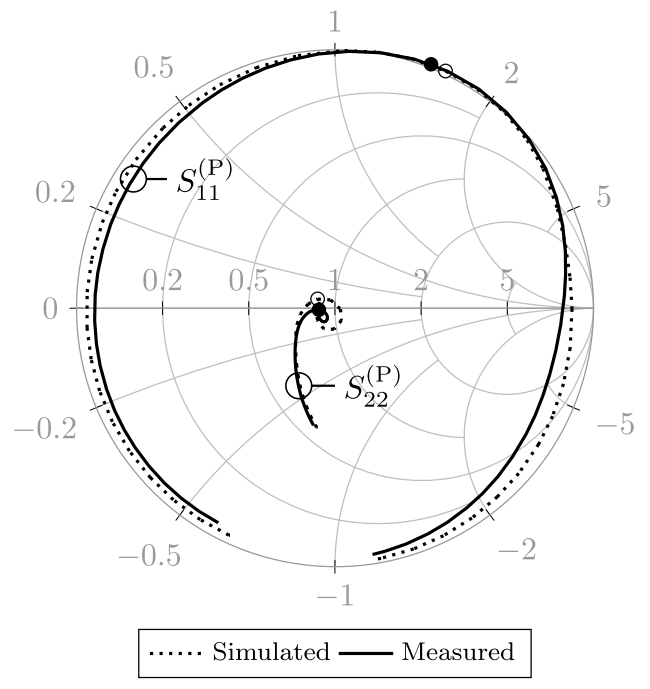

Fig. 6. Simulated and measured input impedance and output impedance of the LNA. Negative resistance occurs from approximately 31-33 MHz.

is $-1.1+j 73 \Omega$ at $32.1 \mathrm{MHz}$, corresponding nicely to the required impedance. The input impedance was adjusted by the tuning capacitors $\mathrm{C} 9 \mathrm{t}$ and $\mathrm{C} 10 \mathrm{t}$, such that the maximum decoupling was achieved. The simulated input impedance is approximately $-1.1+j 78.5 \Omega$. Overall, a good agreement between the simulated and measured impedances is achieved.

To analyze the stability of the LNA, the Edwards-Sinsky stability factor $(\mu)[38]$ is used, as opposed to the Rollet stability factor $(K)$ [39]. This is because the Edwards-Sinsky stability factor, for either the input or output, constitutes the necessary and sufficient condition for stability. The Rollet stability factor requires two equations to ensure stability [22], [39]. If the LNA is designed with a negative input resistance, unconditional stability is not achievable, and the total augmented resistance on the input and output should be larger than zero [21]

$$
\begin{aligned}
R_{\text {in }} & =\operatorname{Re}\left(Z_{\mathrm{S}}+Z^{(\mathrm{P})}\right)>0 \\
R_{\text {out }} & =\operatorname{Re}\left(Z_{\mathrm{L}}+Z^{(\mathrm{OS})}\right)>0
\end{aligned}
$$

where $R_{\mathrm{in}}$ is the augmented input resistance and $R_{\text {out }}$ is the augmented output resistance. The measured and simulated augmented output resistances are above zero over the frequency range from 20 to $40 \mathrm{MHz}$. At lower and higher frequencies, the input impedance has a positive resistance which ensures the stability of the amplifier at these frequencies. The $\mu$ stability factor and the augmented input resistance are plotted in Fig. 7. In the range from approximately 31 to $33 \mathrm{MHz}$, the LNA is conditionally stable, while the LNA is unconditionally stable outside the $31-33-\mathrm{MHz}$ range. To justify the stability of the system, (6) is also examined, showing that with the given combination of coil and matching circuit, the amplifier is indeed stable, since the augmented input resistance is positive. As an additional precaution, the spectrum was measured to check for oscillations-none was observed. Hence, the circuit is stable with the given combination of coil, matching circuit, and LNA.

The simulated and measured gains are seen in Fig. 8. The simulated gain of the LNA is $23 \mathrm{~dB}$, while the measured

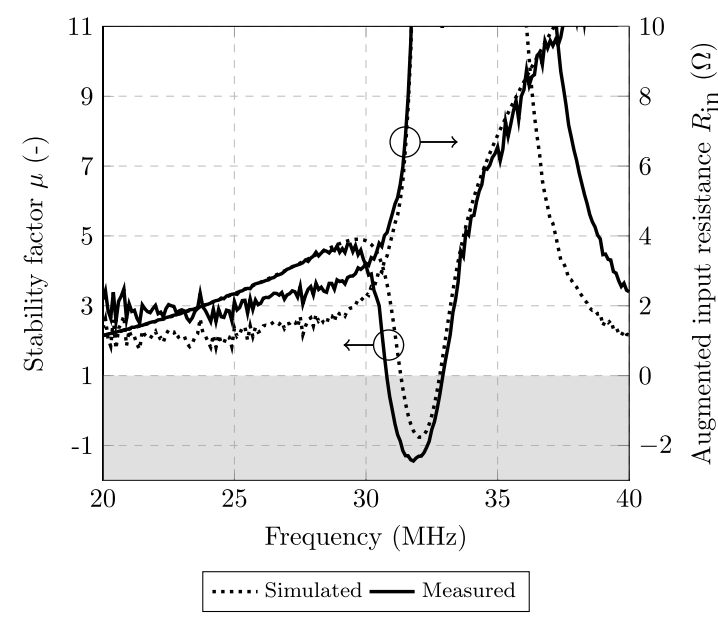

Fig. 7. Left: stability factor of the LNA is shown. Right: input stability resistance is shown. As the stability factor becomes smaller than one, the input stability resistance must be above zero for the LNA to be stable.

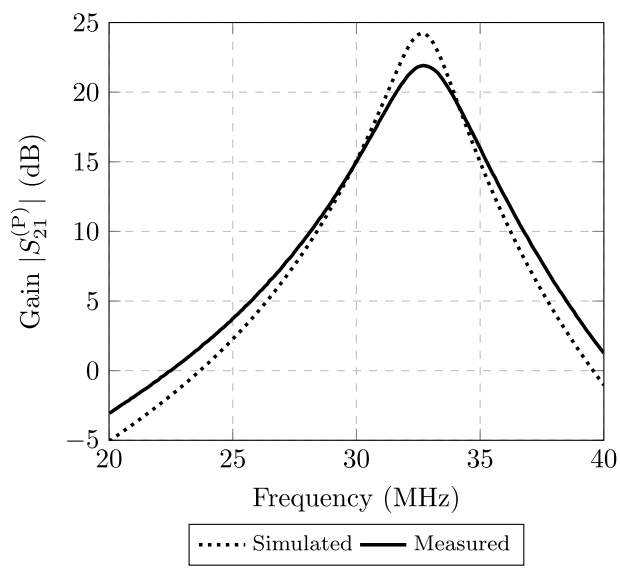

Fig. 8. Simulated and measured gain of the LNA.

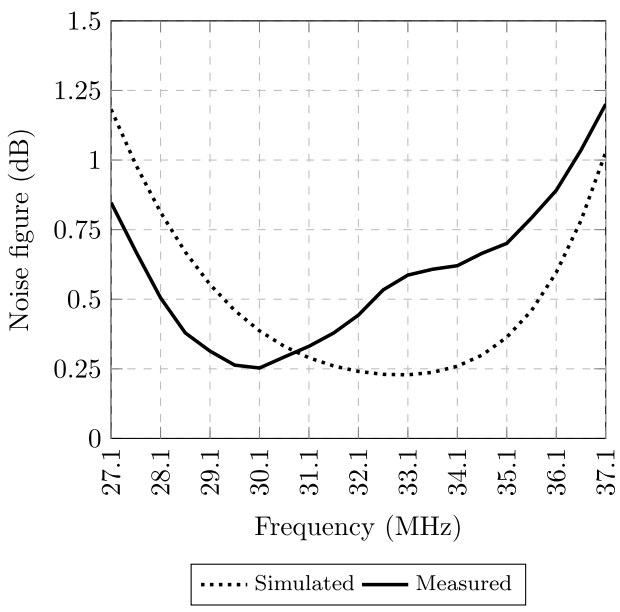

Fig. 9. Simulated and measured noise figure of the LNA.

gain is $22 \mathrm{~dB}$ at $32.1 \mathrm{MHz}$. It is slightly higher than the LNA presented in [27], which showed no SNR degradation compared to the readily available WanTcom LNA. Thus, the gain is acceptable.

At $32.1 \mathrm{MHz}$, the noise figure was simulated and measured at 0.24 and $0.44 \mathrm{~dB}$, respectively. The noise figure is plotted over frequency in Fig. 9. The measured noise figure is slightly 




Fig. 10. Measured 1-dB compression point. Solid line: measured response. Dashed line: linear extrapolation from the low power gain. Dashed dotted line: 1-dB compression point.

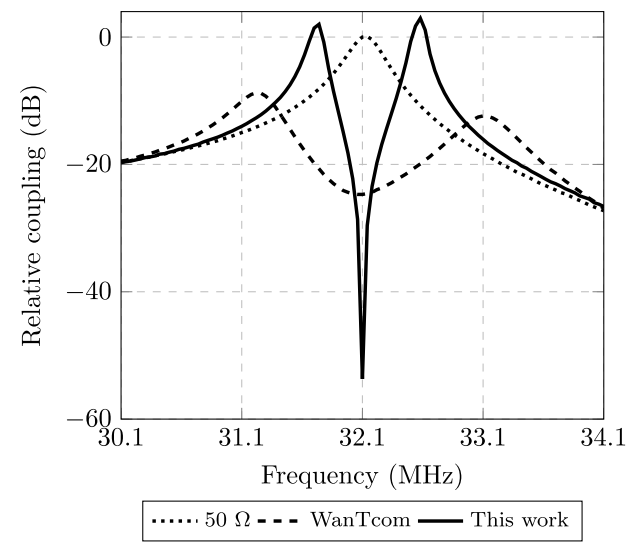

Fig. 11. Relative coupling when utilizing the implemented LNA compared to the WanTcom. Reference value is measured when the matching circuit is terminated by a $50-\Omega$ load.

shifted in frequency. As will be discussed later, the noise figure lies within expected tolerances. In terms of linearity, the $1-\mathrm{dB}$ compression point is measured at $-14.5 \mathrm{dBm}$, shown in Fig. 10. According to [40] and [41], a 1-dB compression point better than $-20 \mathrm{dBm}$ is sufficient. Hence, the LNA complies with linearity requirements.

The implemented LNA draws 27-mA current, from a supply voltage of $3 \mathrm{~V}$ resulting in a power consumption $P^{(\mathrm{P})}=$ $81 \mathrm{~mW}$. The supply voltage can be lowered also to lower the power consumption. A $1.5-\mathrm{V}$ supply was attempted; however, the 1-dB compression point dropped below $-20 \mathrm{dBm}$ which is rather low.

The coupling between two identical array elements loaded with the designed LNAs is shown in Fig. 11, where it is compared to one of the few commercially available MRI LNAs for the $32.1-\mathrm{MHz}$ operation. The coupling is measured by $S_{21}$ using two loop probes of $1-\mathrm{cm}$ diameter separated by $4 \mathrm{~cm}$ connected to a vector network analyzer, as described in [42]-[44]. The reference value is when the matching circuit is terminated by a $50-\Omega$ load. The WanTcom achieves $25-\mathrm{dB}$ decoupling, while the LNA presented in this paper achieves $54 \mathrm{~dB}$ of decoupling. The bandwidth of the decoupling is also decreased.

\section{Discussion}

A detailed analysis of the matching circuit is required to understand why a negative input resistance LNA yields optimal decoupling. It is excluded from a detailed treatment here to keep this paper focused on the design of such an LNA. In brief terms, however, the negative input resistance of the LNA negates losses in the matching network resulting in a higher $Q$-factor of the decoupling circuit.

Overall, a good agreement has been achieved between the simulated and measured results. The coupling between the input and output circuits adds an additional feedback which must be included in simulations to achieve this level of accuracy. In the presented LNA, no shielding has been implemented. Better separation between the input and output circuits is possible by placing a shield around the input and/or output circuits. The primary purpose of the shielding is to lower the noise figure of the LNA even further. This potentially avoids the $0.15-\mathrm{dB}$ increase in noise figure in comparison to the present circuit implementation.

The noise figure simulations and measurements appear slightly as an offset. However, the 95th percentile noise figure uncertainty of the transistor, as specified by the manufacturer, is $0.15 \mathrm{~dB}$. Furthermore, the 95th percentile measurement uncertainty is $0.16 \mathrm{~dB}$. With this in mind, the noise figure measurements and simulations lie within the expected results.

Another aspect, which has a large effect on the measured noise performance, is the noise injected by the voltage supply. The presented design utilizes a linear dropout (LDO) regulator as described in [50]. A switch-mode voltage supply was used in the initial design. The switch-mode supply added approximately $0.2-\mathrm{dB}$ noise compared to the LDO solution.

The concept of flicker noise has not been devoted much attention so far, because it is not an issue with the current transistor when operated at $32.1 \mathrm{MHz}$. However, using the design with a different transistor and/or at a lower frequency, the flicker noise may become an issue. The primary strategy toward flicker noise is to use a transistor technology with a lower corner frequency [25].

The gain is at an acceptable level for this application. However, at higher frequencies, the gain may become an issue due to the lower inherent gain of the transistor. An additional stage can be included to increase the gain. Assuming that the additional second stage has a noise figure of $1 \mathrm{~dB}$, the overall noise figure will degrade by $0.006 \mathrm{~dB}$ according to Friis' equation for cascaded noise figure [51]. This, of course, depends on the gain of the first stage. Assuming that the LNA had a gain of $10 \mathrm{~dB}$, the overall noise figure would be degraded by $0.1 \mathrm{~dB}$. Hence, from a noise perspective, increasing the gain is a simple matter entailing a minor noise impact. However, the additional stage also increases the power consumption.

One of the primary targets of this paper is to demonstrate the maximum achievable decoupling. Hence, having a conditionally stable LNA is acceptable. If less decoupling is tolerated, the stability of the LNA can be increased by adjusting the output circuit accordingly. The required decoupling depends immensely on the design of the coil array. Many designs use overlapping elements for nearest neighbor decoupling and the distance to the next nearest neighbor determines the amount of decoupling required. For most overlapping designs, a decoupling of around $25 \mathrm{~dB}$ is acceptable. For parallel imaging, however, nonoverlapping elements can significantly 
TABLE I

COMPARISON OF LNA DESIGNS FOR MRI

\begin{tabular}{|c|c|c|c|c|c|c|c|}
\hline & Siemens [45], [46] & Cao et. al. [46] & TI [47] & Agile [48] & WanTcom [49] & Johansen et. al. [27] & This work \\
\hline Frequency $(\mathrm{MHz})$ & 128 & 128 & $63 / 128$ & 43 & 32.1 & 32.1 & 32.1 \\
\hline $\mathrm{NF}(\mathrm{dB})$ & 0.71 & 1.02 & 0.6 & 0.4 & 0.7 & 0.75 & 0.44 \\
\hline Gain $(\mathrm{dB})$ & 27 & 32 & 28 & 30 & 28 & 20 & 22 \\
\hline$Z^{(\mathrm{P})}(\Omega)$ & $2.2+j 21$ & $3.6+j 1.6$ & 1.5 & 2 & 3 & $0.4+j 67$ & $-1.1+j 73$ \\
\hline$P_{\mathrm{in}, 1 \mathrm{~dB}}(\mathrm{dBm})$ & -14.5 & -28.5 & 12 & 0 & 9 & -13.8 & -14.5 \\
\hline$P^{(\mathrm{P})}(\mathrm{mW})$ & 196 & 215 & 108 & 152 & 180 & 99 & 81 \\
\hline
\end{tabular}

increase the performance. To collect the most magnetic flux, the nonoverlapping elements need to be placed very close and higher decoupling (depending on separation and shape) is required to not impair both SNR of the individual elements and noise correlation between elements. It is expected that this method is used primarily for parallel imaging coil arrays, where a high degree of decoupling is required.

The bandwidth of the decoupling is fairly narrow, which may be an issue for some MRI sequences having a bandwidth of $50-100 \mathrm{kHz}$. A frequently used sequence for ${ }^{13} \mathrm{C}$ imaging in 3-T MRI scanners is chemical shift imaging (CSI) and it typically requires a bandwidth of $5 \mathrm{kHz}$. Thus, using a CSI sequence, the bandwidth is not an issue. For broadband applications, the presented method can be used if a proper output circuit can be synthesized.

A comparison of different LNA designs for MRI is presented in Table I for, not only $32.1 \mathrm{MHz}$, but higher frequencies as well. The only readily available commercial MRI compatible LNA for $32.1 \mathrm{MHz}$ is the WanTcom. This paper achieves a noise figure of $0.26 \mathrm{~dB}$ better than the WanTcom. For $43 \mathrm{MHz}$, the Agile LNA shows the best noise figure performance of $0.4 \mathrm{~dB}$. The gain of the LNA presented here is lower than the other designs; however, according to [27], this does not pose a problem. As far as the authors are aware, the LNA presented here achieves the best decoupling performance thus far published (when only using the LNA for decoupling). This is because a stable negative input resistance has been realized.

This paper has presented an iterative approach to dissociation LNAs. In terms of practical applicability, we have experienced that an iterative approach is often employed even though the method may principally be analytic. In [26], an analytic method is described which is based on the same theory as this paper and is, at least partly, capable of designing an HRNM LNA. If the analysis of the maximum stable gain in [26] is replaced by an analysis of augmented resistance for stability purposes, it might be possible to change the proposed iterative solution to an analytical. However, because the unconditional stability for negative impedance devices is impossible, the stability of the system will depend on the source and load. Hence, a generalization of the system source and load impedances is required and thus will not truly represent all systems.

The concept of association has not been devoted much attention so far. The transistor used in this paper cannot inherently achieve SNIM. Hence, additional feedback must be added. This is also what other methods require.
However, other methods for SNIM vary only the amount of feedback. From this method, it is possible to combine both feedback and the output circuit impedance. Since bipolar junction-type transistors generally exhibit lower input impedance, they may be better suited for SNIM.

\section{CONCLUSION}

Using noise and S-parameters, a general approach to the LNA design has been proposed. The LNA design spans three cases which we define as design by association (decreasing the mismatch between optimal noise and input impedance), equilibrium (optimal noise matched input while the output is gain matched), and dissociation (high reflection coefficient input impedance while being noise matched). The primary target is an LNA with a negative resistance, inductive input impedance for MRI receive coil arrays. The LNA design achieves a noise figure of $0.44 \mathrm{~dB}$, which is better than existing MRI LNAs at $32.1 \mathrm{MHz}$. The LNA shows a decoupling of $54 \mathrm{~dB}$. The presented method is primarily applicable for designing LNAs with a high input reflection coefficient while exhibiting low noise. However, the method can also be used for SNIM. It is expected that the presented design method and LNA enables new design strategies for MRI receive coil arrays, especially for parallel imaging applications.

\section{APPENDIX \\ Potential BRoAdBAND APPLICATION}

This appendix briefly discusses the potential of applying the design method for applications more broadband than MRI. For this discussion, an ideal common source topology is explored operating from 850 to $1000 \mathrm{MHz}$ (approximately the E-UTRA band 8). The corresponding relative bandwidth is approximately $16 \%$. The biasing and feeding inductors used are ideal (infinitely large and lossless). The same applies for dc blocking capacitors. Using these ideal components, the simulated noise figure, when the source is $50 \Omega$, is maximally $0.21 \mathrm{~dB}$ at $1000 \mathrm{MHz}$. The supply voltage is $3 \mathrm{~V}$, using a biasing voltage of $0.5 \mathrm{~V}$. Hence, when using the ATF58143 transistor, there is no need for additional noise matching according to simulations. Iteratively solving (5) over the frequency range while varying the electrical length of the input transmission line, a solution is found utilizing a passive output circuit. Instead of focusing on a single frequency and plotting the impedance circles, $Z^{(\mathrm{O})}$ is plotted in the Smith Chart over frequency, as seen in Fig. 12. 


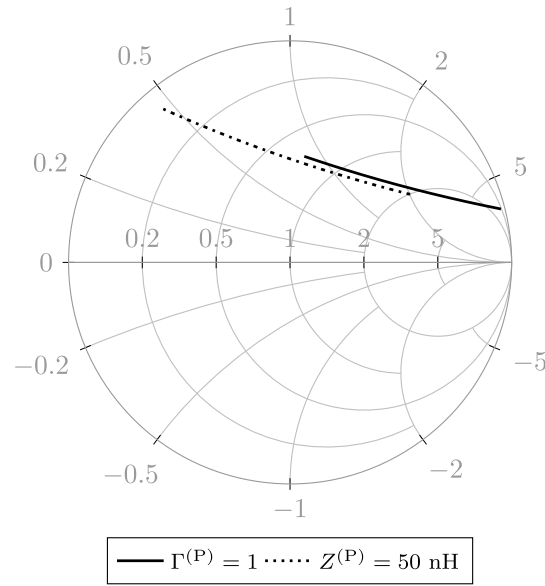

Fig. 12. Simulated required impedance of the output circuit looking toward the load in the frequency range from 850 to $1000 \mathrm{MHz}$ to realize either an ideal instrumentation amplifier (solid) or an ideal inductor (dotted).

For the two examples given in Fig. 12, the required input impedance was either $\Gamma^{(\mathrm{P})}=1$ (solid) or $Z^{(\mathrm{P})}=$ $50 \mathrm{nH}$ (dotted). The electrical length of the transmission line is $100^{\circ}$ at $925 \mathrm{MHz}$, with a characteristic impedance of $50 \Omega$. How to synthesize an output circuit capable of (approximately) providing the required $Z^{(\mathrm{O})}$ will not be discussed in this paper. However, if $Z^{(0)}$ in Fig. 12 can be realized, it is possible to extend the design method to apply for wider bandwidths.

\section{REFERENCES}

[1] X. Cao, D. Zu, X. Zhao, Y. Fan, and J. Gao, "The design of a lownoise preamplifier for MRI," Sci. China Technol. Sci., vol. 54, no. 7, pp. 1766-1770, Jul. 2011.

[2] P. B. Roemer, W. A. Edelstein, and C. E. Hayes, "The NMR phased array," Soc. Magn. Reson. Med., vol. 225, no. 2, pp. 192-225, 1990.

[3] C.-N. Chen and D. I. Hoult, Biomedical Magnetic Resonance Technology, 1st ed. College Park, MD, USA: AIP, 1989.

[4] D. G. Nishimura, Principles of Magnetic Resonance Imaging, 1st ed. 2010. [Online]. Available: https://www.lulu.com

[5] J. Engberg, "Simultaneous input power match and noise optimization using feedback," in Proc. Eur. Microw. Conf., Sep. 1974, pp. 385-389.

[6] L. Boglione, R. D. Pollard, V. Postoyalko, and T. Alam, "Specifications for a linear network simultaneously noise and available-power matched," IEEE Microw. Guided Wave Lett., vol. 6, no. 11, pp. 407-409, Nov. 1996.

[7] L. Boglione, R. D. Pollard, and V. Postoyalko, "BJT feedback LNA with input port simultaneously signal and noise matched," in Proc. 27th EuMC, vol. 2, Sep. 1997, pp. 1052-1057.

[8] L. Boglione, R. D. Pollard, and V. Postoyalko, "Optimum noise-source reflection-coefficient design with feedback amplifiers," IEEE Trans. Microw. Theory Techn., vol. 45, no. 3, pp. 402-407, Mar. 1997.

[9] T.-K. Nguyen, C.-H. Kim, G.-J. Ihm, M.-S. Yang, and S.-G. Lee, "CMOS low-noise amplifier design optimization techniques," IEEE Trans. Microw. Theory Techn., vol. 52, no. 5, pp. 1433-1442, May 2004.

[10] Y. Shim, C.-W. Kim, and S.-G. Lee, "Simultaneous noise and input matched ultra wide band LNA design," Microw. Opt. Tech. Lett., vol. 49, no. 9, pp. 2275-2279, 2007.

[11] R. E. Lehmann and D. D. Heston, "X-band monolithic series feedback LNA," IEEE Trans. Microw. Theory Techn., vol. MTT-33, no. 12, pp. 1560-1566, Dec. 1985.

[12] N. Shiga et al., "X-band MMIC amplifier with pulse-doped GaAs MESFET's," IEEE Trans. Microw. Theory Techn., vol. 39, no. 12, pp. 1987-1994, Dec. 1991.

[13] Y. Tsukahara et al., "A C-band 4-stage low noise miniaturized amplifier using lumped elements," in IEEE MTT-S Int. Microw. Symp. Dig., vol. 3, May 1995, pp. 1125-1128.

[14] S. P. Voinigescu et al., "A scalable high-frequency noise model for bipolar transistors with application to optimal transistor sizing for lownoise amplifier design," IEEE J. Solid-State Circuits, vol. 32, no. 9, pp. 1430-1439, Sep. 1997.
[15] S. Hara, K. Osato, A. Yamada, T. Tsukao, and T. Yoshimasu, "Miniaturized low noise variable MMIC amplifiers with low power consumption for L-band portable communication applications," in IEEE Microw. Millim.-Wave Monolithic Circuits Symp. Dig. Papers, Jun. 1993, pp. $67-70$.

[16] T. Seshita, K. Kawakyu, W. Wakimoto, M. Nagaoka, Y. Kitaura, and N. Uchitomi, "A 2-V operation RF front-end GaAs MMIC for PHS hand-set," in IEEE MTT-S Int. Microw. Symp. Dig., vol. 1, Jun. 1998, pp. $167-170$.

[17] F. Lin, L. Liu, P. S. Kooi, and M. S. Leong, "Design of MMIC LNA for $1.9 \mathrm{GHz}$ CDMA portable communication," in Proc. Microw. Millim. Wave Techn., 1998, pp. 205-208.

[18] K. B. Niclas, "The exact noise figure of amplifiers with parallel feedback and lossy matching circuits," IEEE Trans. Microw. Theory Techn., vol. MTT-30, no. 5, pp. 832-835, May 1982.

[19] K. B. Niclas, "Noise in broad-band GaAs MESFET amplifiers with parallel feedback," IEEE Trans. Microw. Theory Techn., vol. MTT-30, no. 1, pp. 63-70, Jan. 1982.

[20] J. Tajima, Y. Yamao, T. Sugeta, and M. Hirayama, "GaAs monolithic low-power amplifiers with RC parallel feedback (short paper)," IEEE Trans. Microw. Theory Techn., vol. MTT-32, no. 5, pp. 542-544, May 1984.

[21] G. Gonzalez, Microwave Transistor Amplifiers: Analysis and Design, 2nd ed. Upper Saddle River, NJ, USA: Prentice-Hall, 1997.

[22] D. M. Pozar, Microwave Engineering, 3rd ed. Hoboken, NJ, USA: Wiley, 2005.

[23] H. A. Haus et al., "Representation of noise in linear twoports," Proc. IRE, vol. 48, no. 1, pp. 69-74, Jan. 1960.

[24] A. van der Ziel, "Representation of noise in linear two-ports," Proc. IEEE, vol. 57, no. 6, p. 1211, Jun. 1969.

[25] F. A. Levinzon, "Ultra-low-noise high-input impedance amplifier for low-frequency measurement applications," IEEE Trans. Circuits Syst. I, Reg. Papers, vol. 55, no. 7, pp. 1815-1822, Aug. 2008.

[26] W. Ciccognani, P. E. Longhi, S. Colangeli, and E. Limiti, "Constant mismatch circles and application to low-noise microwave amplifier design," IEEE Trans. Microw. Theory Techn., vol. 61, no. 12, pp. 4154-4167, Dec. 2013.

[27] D. H. Johansen, J. D. Sánchez-Heredia, V. Zhurbenko, and J. H. Ardenkjær-Larsen, "Practical aspects of preamplifier designs for 13C imaging," in Proc. Int. Soc. Mag. Reson. Med., 2017.

[28] E. W. Herold, "Negative resistance and devices for obtaining it," Proc. Inst. Radio Eng., vol. 23, no. 10, pp. 1201-1223, Oct. 1935.

[29] J. L. Merrill, "Theory of the negative impedance converter," Bell Syst. Tech. J., vol. 30, no. 1, pp. 88-109, Jan. 1951.

[30] J. G. Linvill, "Transistor negative-impedance converters," Proc. IRE, vol. 41, no. 6, pp. 725-729, 1953.

[31] J. Brownlie, "On the stability properties of a negative impedance converter," IEEE Trans. Circuit Theory, vol. CT-13, no. 1, pp. 98-99, Mar. 1966.

[32] K. Kurokawa, "Power waves and the scattering matrix," IEEE Trans. Microw. Theory Techn., vol. MTT-13, no. 2, pp. 194-202, Mar. 1965.

[33] E. Kreyszig, Advanced Engineering Mathematics, 9th ed. Hoboken, NJ, USA: Wiley, 2006.

[34] D. H. Johansen, J. D. Sánchez-Heredia, V. Zhurbenko, and J. H. Ardenkjær-Larsen, "Towards new vistas in preamplifier design for MRI," in Proc. 12th EuMIC, Oct. 2017, pp. 419-422.

[35] A. Reykowski, S. M. Wright, and J. R. Porter, "Design of matching networks for low noise preamplifiers," Magn. Reson. Med., vol. 33, no. 6, pp. 848-852, 1995.

[36] J. D. Sánchez-Heredia, E. Søvsø, S. Hansen, C. Laustsen, V. Zhurbenko, and J. H. Ardenkjær-larsen, "Low-noise active decoupling circuit and its application to $13 \mathrm{C}$ cryogenic RF coils at 3 T," Tomography, vol. 3, no. 1, pp. 60-66, 2017.

[37] ATF-58143: Low Noise Enhancement Mode Pseudomorphic HEMT in a Surface Mount Plastic Package (Data Sheet), Avago Technol., San Jose, CA, USA, 2012.

[38] M. L. Edwards and J. H. Sinsky, "A new criterion for linear 2-port stability using a single geometrically derived parameter," IEEE Trans. Microw. Theory Techn., vol. 40, no. 12, pp. 2303-2311, Dec. 1992.

[39] J. M. Rollett, "Stability and power-gain invariants of linear twoports," IRE Trans. Circuit Theory, vol. 9, no. 1, pp. 29-32, Mar. 1962.

[40] R. Behin, J. Bishop, and R. M. Henkelman, "Dynamic range requirements for MRI," in Proc. Int. Soc. Mag. Reson. Med., 2005, p. 845.

[41] R. Behin, J. Bishop, and R. M. Henkelman, "Dynamic range requirements for MRI," Concepts Magn. Reson. B, Magn. Reson. Eng., vol. 26 B, no. 1, pp. $28-35,2005$. 
[42] D. I. Hoult and R. E. Richards, "The signal-to-noise ratio of the nuclear magnetic resonance experiment," J. Magn. Reson., vol. 24, no. 1, pp. 71-85, Oct. 1976.

[43] D. I. Hoult and P. C. Lauterbur, "The sensitivity of the zeugmatographic experiment involving human samples," J. Magn. Reson., vol. 34, no. 2, pp. $425-433,1979$.

[44] D. I. Hoult, "The principle of reciprocity in signal strength calculations-A mathematical guide," Concepts Magn. Reson., vol. 12, no. 4, pp. 173-187, 2000

[45] Part Number: 7576312, $128 \mathrm{MHz}$ Preamplifier, Siemens, Munich, Germany.

[46] X. Cao, E. Fischer, J. G. Korvink, O. Gruschke, J. Hennig, and M. Zaitsev, "Design of a $3 \mathrm{~T}$ preamplifier which stability is insensitive to coil loading," J. Magn. Reson., vol. 265, pp. 215-223, Apr. 2016.

[47] TL5500: Low-Noise Pre-Amplifier for MRI (SLAS093C), Texas Instrum., Dallas, TX, USA, 2012.

[48] AMT-AN0064: $40 \mathrm{MHz}$ to $50 \mathrm{MHz}$ Non-Magnetic Low Noise Amplifier, Agile Microw. Technol. Inc., New York, NY, USA.

[49] WMA32C: $32.19 \mathrm{MHz}$ Low Noise Low Impedance Preamplifier, WanTcom Inc., Chanhassen, MN, USA, 2013.

[50] D. H. Johansen, J. D. Sánchez-Heredia, J. R. Petersen, T. K. Johansen, V. Zhurbenko, and J. H. Ardenkjær-Larsen, "Cryogenic preamplifiers for magnetic resonance imaging," IEEE Trans. Biomed. Circuits Syst., vol. 12, no. 1, pp. 202-210, Feb. 2018.

[51] H. T. Friis, "Noise figures of radio receivers," Proc. IRE, vol. 32, no. 7, pp. 419-422, Jul. 1944.

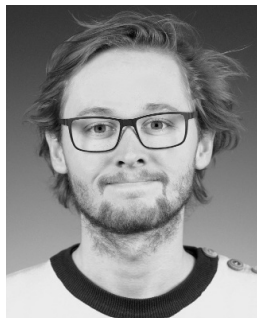

Daniel H. Johansen received the M.Sc. degree (cum laude) in wireless communication systems from Aalborg University, Aalborg, Denmark, in 2013. $\mathrm{He}$ is currently pursuing the Ph.D. degree at the Center for Hyperpolarization in Magnetic Resonance and Electromagnetic Systems, Technical University of Denmark, Kongens Lyngby, Denmark.

He was with RFMD, where he was involved in designing reconfigurable antennas. He was with Sigma Designs, where he was involved in electronics for the Internet of Things. His current research interests include the design and implementation of cryogenic preamplifiers and single and array coils for magnetic resonance imaging.

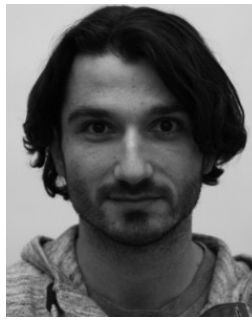

Juan D. Sanchez-Heredia received the M.Sc. degree in telecommunications engineering from the University of Murcia, Murcia, Spain, in 2010, and the Ph.D. degree in telecommunications engineering from the Technical University of Cartagena, Cartagena, Colombia.

In 2014, he joined the Center for Hyperpolarization in Magnetic Resonance, Technical University of Denmark, Kongens Lyngby, Denmark, where he is currently a Post-Doctoral Researcher. His current research interests include the development of multiantenna systems for wireless communications, application of coil arrays to low-gamma nuclei magnetic resource imaging (MRI), low-noise MRI electronics, and high-field MRI coils.

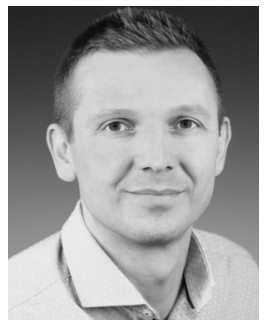

Vitaliy Zhurbenko received the M.Sc. degree in electrical engineering from the Kharkiv National University of Radio Electronics, Kharkiv, Ukraine, in 2001, and the Ph.D. degree in electrical engineering from the Technical University of Denmark (DTU), Kongens Lyngby, Denmark, in 2008 .

In 2005, he joined DTU, where he is currently an Associate Professor. His current research interests include microwave and millimeter-wave sensing for biomedical and security applications, microwave and millimeter-wave components and integrated circuits for instrumentation applications, antennas and passive circuit design and characterization, and magnetic resonance technology.

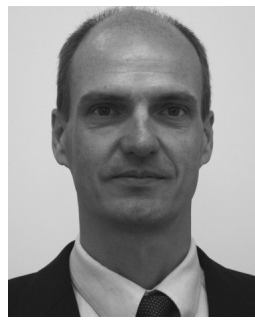

Jan H. Ardenkjær-Larsen received the M.Sc. and $\mathrm{Ph} . \mathrm{D}$. degrees in electrical engineering from the Technical University of Denmark (DTU), Kongens Lyngby, Denmark, in 1991 and 1995, respectively.

$\mathrm{He}$ is currently a Full Professor and the Head of the Center for Magnetic Resonance, DTU, where he is also a Center Leader of the Center for Hyperpolarization in Magnetic Resonance, a Center of Excellence funded by the Danish National Research Foundation. His current research interests include the sensitivity of magnetic resonance imaging and spectroscopy, with the aim of obtaining new, and otherwise inaccessible, information about function and disease at the cellular and molecular levels. 